\title{
Measuring the atmospheric neutrino oscillation parameters and constraining the $3+1$ neutrino model with ten years of ANTARES data
}

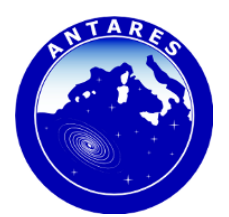

\section{The ANTARES collaboration}

A. Albert, ${ }^{a}$ M. André, ${ }^{b}$ M. Anghinolfi, ${ }^{c}$ G. Anton, ${ }^{d}$ M. Ardid, ${ }^{e}$ J.-J. Aubert, ${ }^{f}$ J. Aublin, ${ }^{g}$ T. Avgitas, ${ }^{g}$ B. Baret, ${ }^{g}$ J. Barrios-Martí, ${ }^{h}$ S. Basa, ${ }^{i}$ B. Belhorma, ${ }^{j}$ V. Bertin, ${ }^{f}$ S. Biagi, ${ }^{k}$ R. Bormuth, ${ }^{l, m}$ J. Boumaaza, ${ }^{n}$ S. Bourret,${ }^{g}$ M.C. Bouwhuis, ${ }^{l}$ H. Brânzaș, ${ }^{o}$ R. Bruijn, ${ }^{l, p}$ J. Brunner, ${ }^{f}$ J. Busto, ${ }^{f}$ A. Capone,,${ }^{q, r}$ L. Caramete, ${ }^{o}$ J. Carr, ${ }^{f}$ S. Celli, ${ }^{q, r, s}$ M. Chabab, ${ }^{t}$ R. Cherkaoui El Moursli, ${ }^{n}$ T. Chiarusi, ${ }^{u}$ M. Circella, ${ }^{v}$ A. Coleiro, ${ }^{h, g}$ M. Colomer,${ }^{g, h}$ R. Coniglione, ${ }^{k}$ H. Costantini, ${ }^{f}$ P. Coyle, ${ }^{f}$ A. Creusot, ${ }^{g}$ A.F. Díaz, ${ }^{w}$ A. Deschamps, ${ }^{x}$ C. Distefano, ${ }^{k}$ I. Di Palma, ${ }^{q, r}$ A. Domi, ${ }^{c, y}$ R. Donà, ${ }^{u}$ C. Donzaud, ${ }^{g, z}$ D. Dornic, ${ }^{f}$ D. Drouhin, ${ }^{a}$ T. Eberl, ${ }^{d}$ I. El Bojaddaini, ${ }^{a} a$ N. El Khayati, ${ }^{n}$ D. Elsässer, ${ }^{a b}$ A. Enzenhöfer, ${ }^{d, f}$ A. Ettahiri, ${ }^{n}$ F. Fassi, ${ }^{n}$ P. Fermani,,${ }^{q, r}$ G. Ferrara, ${ }^{k}$ L. Fusco, ${ }^{g, a c}$ P. Gay, ${ }^{a d, g}$ H. Glotin, ${ }^{a e}$ R. Gozzini, ${ }^{h}$ T. Grégoire, ${ }^{g}$ R. Gracia Ruiz, ${ }^{a}$ K. Graf, ${ }^{d}$ S. Hallmann, ${ }^{d}$ H. van Haren, ${ }^{a f}$ A.J. Heijboer, ${ }^{l}$ Y. Hello, ${ }^{x}$ J.J. Hernández-Rey, ${ }^{h}$ J. HößI, ${ }^{d}$ J. Hofestädt, ${ }^{d}$ G. Illuminati, ${ }^{h}$ C.W. James, ${ }^{a g, a h}$ M. de Jong, ${ }^{l, m}$ M. Jongen, ${ }^{l}$ M. Kadler, ${ }^{a b}$ O. Kalekin, ${ }^{d}$ U. Katz, ${ }^{d}$ N.R. Khan-Chowdhury, ${ }^{h}$ A. Kouchner, ${ }^{g, a i}$ M. Kreter, ${ }^{a b}$ I. Kreykenbohm, ${ }^{a j}$ V. Kulikovskiy, ${ }^{c, a k}$ C. Lachaud ${ }^{g}$ R. Lahmann, ${ }^{d}$ R. Le Breton, ${ }^{g}$ D. Lefèvre, ${ }^{a l}$ E. Leonora, ${ }^{a m}$ G. Levi, ${ }^{u, a c}$ M. Lincetto, ${ }^{f}$ M. Lotze, ${ }^{h}$ S. Loucatos, ${ }^{a n, g}$ G. Maggi, ${ }^{f}$ M. Marcelin, ${ }^{i}$ A. Margiotta, ${ }^{u, a c}$ A. Marinelli, ${ }^{a, a p}$ J.A. Martínez-Mora, ${ }^{e}$ R. Mele, ${ }^{a q, a r}$ K. Melis, ${ }^{l, p}$ P. Migliozzi, ${ }^{a q}$ A. Moussa, ${ }^{a}{ }^{a}$ S. Navas, ${ }^{a s}$ E. Nezri, ${ }^{i}$ C. Nielsen, ${ }^{g}$ A. Nuñez, ${ }^{f, i}$ M. Organokov, ${ }^{a}$ G.E. Păvălaș, ${ }^{o}$ C. Pellegrino, ${ }^{u, a c}$ M. Perrin-Terrin, ${ }^{f}$ P. Piattelli, ${ }^{k}$ V. Popa, ${ }^{o}$ T. Pradier, ${ }^{a}$ L. Quinn, ${ }^{f}$ C. Racca, ${ }^{a t}$ N. Randazzo, ${ }^{a m}$ G. Riccobene, ${ }^{k}$ A. Sánchez-Losa, ${ }^{v}$ A. Salah-Eddine, ${ }^{t}$ I. Salvadori, ${ }^{f, 1}$ D.F.E. Samtleben, ${ }^{l, m}$ M. Sanguineti, ${ }^{c, y}$ P. Sapienza, ${ }^{k}$ F. Schüssler, ${ }^{a n}$ M. Spurio, ${ }^{u, a c}$

\footnotetext{
${ }^{1}$ Corresponding author.
} 
Th. Stolarczyk, ${ }^{a n}$ M. Taiuti, ${ }^{c, y}$ Y. Tayalati, ${ }^{n}$ T. Thakore, ${ }^{h}$ A. Trovato, ${ }^{k}$ B. Vallage, ${ }^{a n, g}$ V. Van Elewyck, ${ }^{g, a i}$ F. Versari, ${ }^{u, a c}$ S. Viola, ${ }^{k}$ D. Vivolo, ${ }^{a q, a r}$ J. Wilms, ${ }^{a j}$ D. Zaborov, ${ }^{f}$ J.D. Zornoza ${ }^{h}$ and J. Zúñiga ${ }^{h}$

${ }^{a}$ Université de Strasbourg, CNRS, IPHC UMR 7178, F-67000 Strasbourg, France

${ }^{b}$ Technical University of Catalonia, Laboratory of Applied Bioacoustics, Rambla Exposició, 08800 Vilanova i la Geltrú, Barcelona, Spain

${ }^{c}$ INFN — Sezione di Genova, Via Dodecaneso 33, 16146 Genova, Italy

${ }^{d}$ Friedrich-Alexander-Universität Erlangen-Nürnberg, Erlangen Centre for Astroparticle Physics, Erwin-Rommel-Str. 1, 91058 Erlangen, Germany

${ }^{e}$ Institut d'Investigació per a la Gestió Integrada de les Zones Costaneres (IGIC), Universitat Politècnica de València, c/ Paranimf 1, 46730 Gandia, Spain

${ }^{f}$ Aix Marseille University, CNRS/IN2P3, CPPM, Marseille, France

${ }^{g}$ APC, Université Paris Diderot, CNRS/IN2P3, CEA/Irfu,

Observatoire de Paris, Sorbonne Paris Cité, France

${ }^{h}$ IFIC - Instituto de Física Corpuscular (CSIC - Universitat de València),

c/ Catedrático José Beltrán 2, E-46980 Paterna, Valencia, Spain

${ }^{i}$ LAM - Laboratoire d'Astrophysique de Marseille, Pôle de l'Étoile Site de Château-Gombert, Rue Frédéric Joliot-Curie 38, 13388 Marseille Cedex 13, France

${ }^{j}$ National Center for Energy Sciences and Nuclear Techniques,

B.P. 1382, R.P. 10001 Rabat, Morocco

${ }^{k}$ INFN - Laboratori Nazionali del Sud (LNS), Via S. Sofia 62, 95123 Catania, Italy

${ }^{l}$ Nikhef, Science Park, Amsterdam, The Netherlands

${ }^{m}$ Huygens-Kamerlingh Onnes Laboratorium, Universiteit Leiden, The Netherlands

${ }^{n}$ University Mohammed V in Rabat, Faculty of Sciences,

4 av. Ibn Battouta, B.P. 1014, R.P. 10000 Rabat, Morocco

${ }^{\circ}$ Institute of Space Science, RO-077125 Bucharest, Măgurele, Romania

${ }^{p}$ Universiteit van Amsterdam, Instituut voor Hoge-Energie Fysica,

Science Park 105, 1098 XG Amsterdam, The Netherlands

${ }^{q}$ INFN - Sezione di Roma, P.le Aldo Moro 2, 00185 Roma, Italy

${ }^{r}$ Dipartimento di Fisica dell'Università La Sapienza, P.le Aldo Moro 2, 00185 Roma, Italy

${ }^{s}$ Gran Sasso Science Institute, Viale Francesco Crispi 7, 00167 L'Aquila, Italy

${ }^{t}$ LPHEA, Faculty of Science - Semlali, Cadi Ayyad University, P.O.B. 2390, Marrakech, Morocco

${ }^{u}$ INFN - Sezione di Bologna, Viale Berti-Pichat 6/2, 40127 Bologna, Italy

${ }^{v}$ INFN - Sezione di Bari, Via E. Orabona 4, 70126 Bari, Italy

${ }^{w}$ Department of Computer Architecture and Technology/CITIC, University of Granada, 18071 Granada, Spain

${ }^{x}$ Géoazur, UCA, CNRS, IRD, Observatoire de la Côte d'Azur, Sophia Antipolis, France

${ }^{y}$ Dipartimento di Fisica dell'Università, Via Dodecaneso 33, 16146 Genova, Italy

${ }^{z}$ Université Paris-Sud, 91405 Orsay Cedex, France

${ }^{a a}$ University Mohammed I, Laboratory of Physics of Matter and Radiations,

B.P. 717, Oujda 6000, Morocco

${ }^{a b}$ Institut für Theoretische Physik und Astrophysik, Universität Würzburg,

Emil-Fischer Str. 31, 97074 Würzburg, Germany

${ }^{a c}$ Dipartimento di Fisica e Astronomia dell'Università, Viale Berti Pichat 6/2, 40127 Bologna, Italy 
${ }^{a d}$ Laboratoire de Physique Corpusculaire, Clermont Université, Université Blaise Pascal, CNRS/IN2P3, BP 10448, F-63000 Clermont-Ferrand, France

${ }^{a e}$ LIS, UMR Université de Toulon, Aix Marseille Université, CNRS, 83041 Toulon, France

${ }^{a f}$ Royal Netherlands Institute for Sea Research (NIOZ) and Utrecht University, Landsdiep 4, 1797 SZ 't Horntje (Texel), The Netherlands

${ }^{a g}$ International Centre for Radio Astronomy Research - Curtin University, Bentley, WA 6102, Australia

${ }^{a h}$ ARC Centre of Excellence for All-sky Astrophysics (CAASTRO), Australia

${ }^{a i}$ Institut Universitaire de France, 75005 Paris, France

${ }^{a j}$ Dr. Remeis-Sternwarte and ECAP, Friedrich-Alexander-Universität Erlangen-Nürnberg, Sternwartstr. 7, 96049 Bamberg, Germany

${ }^{a k}$ Moscow State University, Skobeltsyn Institute of Nuclear Physics, Leninskie gory, 119991 Moscow, Russia

${ }^{a l}$ Mediterranean Institute of Oceanography (MIO), Aix-Marseille University, 13288, Marseille, Cedex 9, France;

Université du Sud Toulon-Var, CNRS-INSU/IRD UM 110, 83957, La Garde Cedex, France ${ }^{a m}$ INFN - Sezione di Catania, Via S. Sofia 64, 95123 Catania, Italy

${ }^{a n}$ IRFU, CEA, Université Paris-Saclay, F-91191 Gif-sur-Yvette, France

${ }^{a o}$ INFN - Sezione di Pisa, Largo B. Pontecorvo 3, 56127 Pisa, Italy

${ }^{a p}$ Dipartimento di Fisica dell'Università, Largo B. Pontecorvo 3, 56127 Pisa, Italy

${ }^{a q}$ INFN - Sezione di Napoli, Via Cintia, 80126 Napoli, Italy

${ }^{a r}$ Dipartimento di Fisica dell'Università Federico II di Napoli, Via Cintia, 80126 Napoli, Italy

${ }^{a s}$ Departamento de Física Teórica y del Cosmos \&3 C.A.F.P.E., University of Granada, 18071 Granada, Spain

${ }^{a t}$ GRPHE - Université de Haute Alsace - Institut Universitaire de Technologie de Colmar, Rue du Grillenbreit 34, BP 50568-68008 Colmar, France

E-mail: salvadori@cppm.in2p3.fr

ABSTRACT: The ANTARES neutrino telescope has an energy threshold of a few tens of $\mathrm{GeV}$. This allows to study the phenomenon of atmospheric muon neutrino disappearance due to neutrino oscillations. In a similar way, constraints on the $3+1$ neutrino model, which foresees the existence of one sterile neutrino, can be inferred. Using data collected by the ANTARES neutrino telescope from 2007 to 2016, a new measurement of $\Delta m_{32}^{2}$ and $\theta_{23}$ has been performed - which is consistent with world best-fit values - and constraints on the $3+1$ neutrino model have been derived.

Keywords: Neutrino Detectors and Telescopes (experiments), Oscillation

ARXIV EPRINT: 1812.08650 


\section{Contents}

1 Introduction 1

2 The ANTARES neutrino telescope 4

3 ANTARES data and Monte Carlo samples 4

4 Event reconstruction $\quad 5$

5 Analysis 6

5.1 Treatment of systematics for the standard oscillation analysis 9

5.2 Treatment of systematics for the sterile oscillation analysis 11

6 Results 12

$\begin{array}{lll}\text { 6.1 Results for the standard oscillation analysis } & 12\end{array}$

$\begin{array}{lll}6.2 & \text { Results for the sterile oscillation analysis } & 13\end{array}$

$\begin{array}{lll}7 & \text { Conclusions } & 16\end{array}$

$\begin{array}{ll}\text { A Sterile neutrinos and matter effects } & 16\end{array}$

\section{Introduction}

Neutrino oscillations arise from the mixing between flavour $\left(\nu_{e}, \nu_{\mu}, \nu_{\tau}\right)$ and mass $\left(\nu_{1}, \nu_{2}, \nu_{3}\right)$ eigenstates. The mixing parameters of the Pontecorvo-Maki-Nakagawa-Sakata matrix [1-3] (PMNS) and the differences between the mass eigenvalues regulate the oscillation probability.

Neutrino oscillations have been detected by a variety of experiments, studying solar as well as atmospheric neutrinos, but also neutrinos produced from nuclear reactors and particle accelerators. For a comprehensive review see [4].

Atmospheric neutrinos are produced through the interaction of cosmic rays with nuclei in the Earth's atmosphere. Their flux spans many orders of magnitude in energy, from GeV to hundreds of $\mathrm{TeV}$. Being isotropic to first order, it allows to investigate a large range of baselines on the Earth's surface, from $\sim 10 \mathrm{~km}$ of vertically down-going to $\sim 10^{4} \mathrm{~km}$ of vertically up-going neutrinos.

In this paper the muon disappearance channel $\left(P_{\nu_{\mu} \rightarrow \nu_{\mu}}\right)$ is studied. The vacuum survival probability for a muon neutrino of energy $E$ interacting at a distance $L$ from its creation point is given by:

$$
P_{\nu_{\mu} \rightarrow \nu_{\mu}}=1-4 \sum_{j>i}\left|U_{\mu j}\right|^{2}\left|U_{\mu i}\right|^{2} \sin ^{2}\left(\frac{\Delta m_{j i}^{2} L}{4 E}\right) \sim 1-4\left|U_{\mu 3}\right|^{2}\left(1-\left|U_{\mu 3}\right|^{2}\right) \sin ^{2}\left(\frac{\Delta m_{32}^{2} L}{4 E}\right),
$$


where $U_{\mu i}, U_{\mu j}$ are elements of the PMNS matrix $U$, and $\Delta m_{j i}^{2}=m_{j}^{2}-m_{i}^{2}$ are the mass splittings between two mass eigenstates. The rightmost term describes the "single $\Delta m^{2}$ dominance" approximation, relevant in the energy domain considered for this analysis. Here the $\nu_{\mu}$ survival probability depends only on $U_{\mu 3}=\sin \theta_{23} \cos \theta_{13}$ and $\Delta m_{32}^{2}$. For a vertically up-going atmospheric $\nu_{\mu}$, the first minimum of the survival probability described in equation (1.1) is reached at energies of $\sim 25 \mathrm{GeV}$. The formalism given in eq. (1.1) is further modified by matter effects [5-7] as the neutrinos propagate through the Earth. Throughout the paper, oscillation probabilities are calculated with the OscProb package [8] which treats matter effects for an arbitrary number of neutrino families numerically without approximations.

The ANTARES neutrino telescope [9] has been designed and optimised for the exploration of the high-energy Universe by using neutrinos as cosmic probes. However, its energy threshold of about $20 \mathrm{GeV}$ is sufficient, even if at the edge, to be sensitive to the first atmospheric oscillation minimum, making also the study of neutrino oscillations possible. As neutrinos and antineutrinos are indistinguishable on an event-by-event basis in neutrino telescopes, in the following muon (electron) neutrinos are refered to the sum of contributions from both neutrinos and antineutrinos.

A previous analysis of ANTARES data, covering the data acquisition period from 2007 to 2010, represented the first study of this kind performed by a neutrino telescope, and measured the atmospheric neutrino oscillation parameters, $\Delta m_{32}^{2}$ and $\theta_{23}$ [10]. In the present work, data collected during 10 years have been studied with a new analysis chain that also includes a more comprehensive treatment of various systematic effects.

Despite the fact that neutrino oscillation is a well established phenomenon, some observed experimental anomalies, such as the ones reported by the LSND [11] and MiniBooNE [12] collaborations, seem to indicate a deviation from the standard 3-flavour picture. These discrepancies could be partially explained by introducing in the model an additional neutrino state. However, since the number of weakly interacting families of light neutrinos is limited to three by the LEP results [13], the additional neutrinos have to be sterile, i.e., they do not undergo weak interactions.

The $3+1$ neutrino model foresees the existence of one sterile neutrino in addition to the three standard ones. A choice has to be made, how to extend the mixing matrix $U$ from three to four families. In this analysis the convention from [14] (see "supplementary materials") is adopted: $U_{3+1}=R_{34} R_{24} R_{14} R_{23} R_{13} R_{12}$ where $R_{i j}$ is the rotation matrix for angle $\theta_{i j}$. If $j-i>1, R_{i j}$ also contains a CP-violating phase, $\delta_{i j}$. Six new real mixing parameters have to be accounted for: three new mixing angles, $\theta_{14}, \theta_{24}$ and $\theta_{34}$, a new mass splitting, $\Delta m_{41}^{2}$, and two new phases, $\delta_{14}$ and $\delta_{24}$. In line with other analyses of sterile neutrinos in the muon disappearance channel [14-16], $\theta_{14}=0$ is assumed, which also eliminates any dependency on $\delta_{14}$.

Even though a sterile neutrino does not interact as the active flavours, its presence would still modify the oscillation pattern of the standard neutrinos, due to the fact that the standard neutrino flavours could oscillate into these additional sterile species. In particular, 


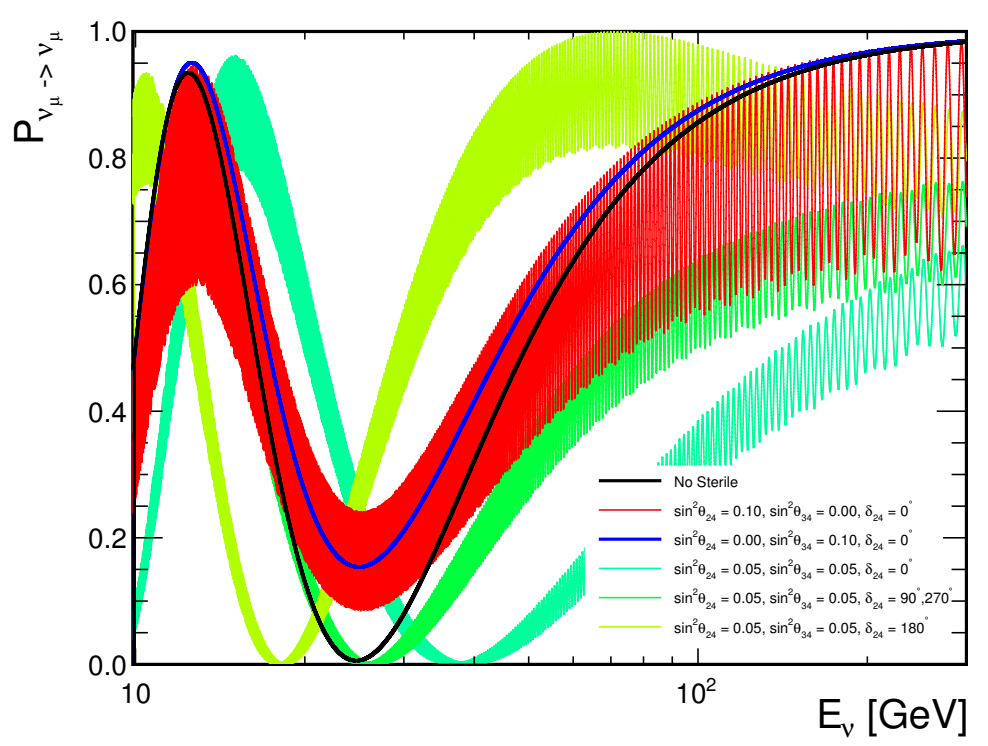

Figure 1. Survival probability of vertically up-going $\nu_{\mu}$ as a function of neutrino energy (calculated with [8]) for different values of mixing angles $\theta_{24}, \theta_{34}$ and $\delta_{24}$ with $\Delta m_{41}^{2}=0.5 \mathrm{eV}^{2}, \Delta m_{31}^{2}=$ $2.5 \cdot 10^{-3} \mathrm{eV}^{2}$ and $\sin ^{2} 2 \theta_{23}=1$.

for up-going $\nu_{\mu}$ in the energy range of $20-100 \mathrm{GeV}$, non-zero values of $U_{\mu 4}$ and $U_{\tau 4}$ with

$$
\begin{aligned}
& U_{\mu 4}=e^{-i \delta_{24}} \sin \theta_{24}, \\
& U_{\tau 4}=\sin \theta_{34} \cos \theta_{24} .
\end{aligned}
$$

can lead to distortions in their survival probability. This is illustrated in figure 1 which shows the $\nu_{\mu}$ survival probability for maximal mixing of $\theta_{23}$ and different combinations of the mixing parameters $\theta_{24}, \theta_{34}$ and $\delta_{24}$. If only $\theta_{34}$ is non-zero, the survival probability of $\nu_{\mu}$ with respect to the non-sterile hypothesis is only modified close to the first oscillation minimum. The case of both $\theta_{24}$ and $\theta_{34}$ being non-zero leads instead to a significant shift of the first oscillation minimum in energy (depending on $\delta_{24}$ ) and modifies the event rate up to energies of few hundred $\mathrm{GeV}$, easily accessible with ANTARES. The fast wiggles due to $\Delta m_{41}^{2}=0.5 \mathrm{eV}^{2}$ will be smeared out by detector resolution effects, therefore no sensitivity to this parameter is expected. The surprisingly strong effect of $\delta_{24}$ on the $\nu_{\mu}$ survival probability, neglected in all similar analyses so far, is further detailed in the appendix.

Since the effect of an additional sterile neutrino would be visible in the same energy and zenith range as the $\nu_{\mu}$ disappearance, the same analysis chain and data sample can be exploited to constrain the $3+1$ neutrino model parameters. In this paper, the results of an investigation aiming to constrain the mixing angles $\theta_{24}$ and $\theta_{34}$ of the $3+1$ neutrino model are also reported.

The paper is organised as follows: in section 2 the ANTARES neutrino telescope is briefly described and its detection principle is illustrated; the ANTARES data sample as well as the Monte Carlo (MC) chain are presented in section 3, while the event reconstruction is discussed in section 4 . Section 5 is dedicated to the event selection and the 
minimisation procedure. The results are presented in section 6 , while conclusions are given in section 7 .

\section{The ANTARES neutrino telescope}

The ANTARES neutrino telescope is located in the Mediterranean Sea, $40 \mathrm{~km}$ off the coast of Toulon, France, at a mooring depth of about $2475 \mathrm{~m}$. The detector was completed in 2008. ANTARES is composed of 12 detection lines, each one equipped with 25 storeys of 3 optical modules (OMs), except line 12 with only 20 storeys of OMs, for a total of 885 OMs. The horizontal spacing among the lines is $\sim 60 \mathrm{~m}$, while the vertical spacing between the storeys is $14.5 \mathrm{~m}$. Each OM hosts a 10-inch photomultiplier tube (PMT) from Hamamatsu [17], whose axis points $45^{\circ}$ downwards. All signals from the PMTs that pass a threshold of 0.3 single photoelectrons (hits) are digitised and sent to the shore station [18, 19]. The on-shore trigger system [20] performs a hit selection based on causality relations and builds events under the hypothesis that the selected hits originate from Cherenkov radiation induced by relativistic charged particles as they are produced in neutrino interactions close to the ANTARES instrumented volume.

The main sources of optical background registered by the ANTARES PMTs are represented by Cherenkov light from decay products of the radioactive isotope ${ }^{40} \mathrm{~K}$, naturally present in sea-water, by light emitted through bioluminescence by living organisms, and by energetic atmospheric muons, which can penetrate deeply under the sea and reach the detector from above.

\section{ANTARES data and Monte Carlo samples}

ANTARES data collected from 2007 to 2016 have been considered in the analysis. After excluding data acquired under adverse conditions, a total of 2830 days of live time has been evaluated.

The aim of the MC production is to reproduce in the most realistic way the events expected at the detector, as well as the response of the apparatus when recording these events. In order to account for changes of the environmental conditions, as well as for the different operational status of the detector and its components over time, a run-byrun MC approach is applied [21]. A typical run lasts few hours. Several time dependent conditions are taken from real data and applied to the run-by-run MC. First, temporarily or permanently non-operational OMs are masked in the simulation. Secondly, background light conditions, which might vary due to bioluminescence, are measured every $104 \mathrm{~ms}$ for each individual OM. These samples are directly used as input for the background light simulation. Thirdly, individual OM efficiencies are considered, as calculated on an approximately weekly basis from ${ }^{40} \mathrm{~K}$ coincidence rates [22]. Finally, the acoustics based position calibration, performed every few minutes, is applied. All these detailed inputs assure an authentic description of the detector response for each individual run. Remaining uncertainties are small and can be handled as global parameters which are discussed below. They are included in the analysis as systematic uncertainties. 
Neutrino interactions of all flavours have been simulated with the GENHEN [23] package, developed inside the ANTARES Collaboration. It allows to reproduce neutrino interactions in the $\mathrm{GeV}$ to multi-PeV energy range. $\mathrm{MC}$ neutrino events can be weighted to reproduce different physical expectations. For atmospheric neutrinos with $E_{\nu} \in[20-$ $100] \mathrm{GeV}$, a MC sample almost three hundreds times larger than the data sample is available. The model by Honda et al. [24] for the Fréjus site is used in this work.

Even though the sub-marine location of ANTARES provides a good shielding against atmospheric muons, still a large amount of them will reach the detector. The event generator used in ANTARES to simulate atmospheric muons is MUPAGE [25]; the energy and angular distributions, as well as the multiplicity of muons propagating in sea water are parameterised. The contribution from this background is also evaluated from the data itself.

Particle propagation and Cherenkov light production are simulated using a GEANTbased [26] package [23], which takes into account all relevant physics processes and computes the probability that photons emitted by a particle reach the OM surface, producing a hit. Finally the detector response is simulated, including the digitisation and filtering of hits. At this stage a realistic optical noise is added on each OM for each data acquisition run of the detector, and the time evolution of the detector configuration is accounted for as described above.

\section{Event reconstruction}

Charged-current (CC) interactions of muon neutrinos produce a muon propagating through the detector and inducing Cherenkov light. They are identified as track-like events. The event reconstruction and selection used in the analysis have been optimised to select such events. On the other hand, $\nu_{e} \mathrm{CC}$ interactions, as well as neutral-current interactions (NC) of all flavours produce hadronic showers. In the case of $\nu_{e} \mathrm{CC}$ interactions an electromagnetic shower is produced as well. Moreover, $\nu_{\tau}$ CC events can be produced as the result of $\nu_{\mu} \rightarrow \nu_{\tau}$ oscillations with and without muons in the final state. All these events constitute an additional source of background for this study.

Events have been reconstructed using two different algorithms, described in detail in $[27,28]$. In the following discussion these algorithms will be referred to as method $\mathcal{A}$ and method $\mathcal{B}$, respectively. Both are optimised for events induced by $\mathrm{GeV}$-scale $\nu_{\mu} \mathrm{CC}$ interactions. In method $\mathcal{A}$ a hit selection, based on time and spatial coincidences of hits, is applied and a $\chi^{2}$-fit is performed in order to find the best track. Events can have a singleline topology (SL), if all the selected hits have been recorded in the same detector line, or a multi-line topology (ML), when hits belong to OMs of different lines. Method $\mathcal{B}$ consists of a chain of fits, aimed to improve at each step the track estimation. Starting from a hit selection, a first prefit, based on a directional scan with a large number of isotropically distributed directions, is performed. The best 9 directions are used as starting points for the final likelihood $(\log \mathcal{L})$ fit.

Once the muon track has been reconstructed, its length, $L_{\mu}$, is computed. This is done, for ML events, by projecting back to the track the first and last selected hit. For SL events, 
since a vertex estimation is not possible due to the lack of azimuth information, the track length is estimated from the z-coordinates of the uppermost and lowermost storey which have recorded the selected hits and taking into account the reconstructed zenith angle.

The muon energy estimation is based on the fact that muons in the few-GeV energy range can be treated as minimum ionising particles, and their energy can be estimated from their track length $L_{\mu}$ :

$$
E_{\text {reco }}=L_{\mu} \times 0.24 \mathrm{GeV} / \mathrm{m} \text {, }
$$

where the factor $0.24 \mathrm{GeV} / \mathrm{m}$ represents the energy loss of muons in sea water in the energy range of $10-100 \mathrm{GeV}$ [29]. This quantity is used in the following as estimator for the neutrino energy. The energy resolution of fully contained muons is dominated by the spacing of the detector elements and is found to be around $5 \mathrm{GeV}$. For muons leaving the detector only a lower limit for their energy can be derived, corresponding to their visible length inside the instrumented volume. More details on the muon energy resolution can be found in [10].

\section{Analysis}

To achieve the best sensitivity to the measurement of the oscillation parameters, a set of quality criteria has been applied. The selection of $\nu_{\mu} \mathrm{CC}$ events has been optimised by performing a preliminary Monte Carlo (MC) sensitivity study, before applying the whole analysis chain to data.

The main parameter on which the selection is based is the reduced $\chi^{2}$ for method $\mathcal{A}$ and the $\log \mathcal{L}$ for method $\mathcal{B}$. Events reconstructed by method $\mathcal{A}$ and passing the corresponding event selection are kept. The events discarded by this procedure are further reconstructed by method $\mathcal{B}$; they are kept in the analysed sample if the corresponding selection criteria are passed. Only events which are reconstructed as up-going are used in the following. A minimum number of five storeys with selected hits is required, in order to minimise the background induced by atmospheric muons.

In figure 2 the distribution of the $\mathrm{MC}$ true neutrino energy, $\mathrm{E}_{\mathrm{T}}$, for selected $\nu_{\mu} \mathrm{CC}$ events is shown. For the histogram with the solid line no neutrino oscillations are assumed, while the dashed one refers to a 2-flavour oscillation scenario with maximal mixing and $\Delta m_{32}^{2}=2.46 \times 10^{-3} \mathrm{eV}^{2}$. As can be seen, atmospheric neutrino oscillations affect the expected event distribution for $\mathrm{E}_{\mathrm{T}} \lesssim 100 \mathrm{GeV}$. About 7590 well-reconstructed $\nu_{\mu} \mathrm{CC}$ events are expected in a live time of 2830 days when oscillations are neglected. Roughly one half of these events are reconstructed with method $\mathcal{A}(\mathrm{ML})$, while methods $\mathcal{A}$ (SL) and $\mathcal{B}$ both contribute with approximately $25 \%$ to this event sample. Further, $\sim 40 \nu_{e}$ $\mathrm{CC}$ events are selected. Oscillations reduce the number of expected events by $\sim 720$ events. This reduction is dominantly seen in the $\mathcal{A}(\mathrm{SL})$ sample $(\sim 60 \%)$ which contains the lowest energetic and most vertical events, while the other two reconstruction methods contribute each about $20 \% . \nu_{\tau}$ CC events reduce this oscillation signal by $\sim 20$ events, taking into account the energy-dependent cross section ratio $\sigma\left(\nu_{\tau} \mathrm{CC}\right) / \sigma\left(\nu_{\mu} \mathrm{CC}\right.$ ) (about 


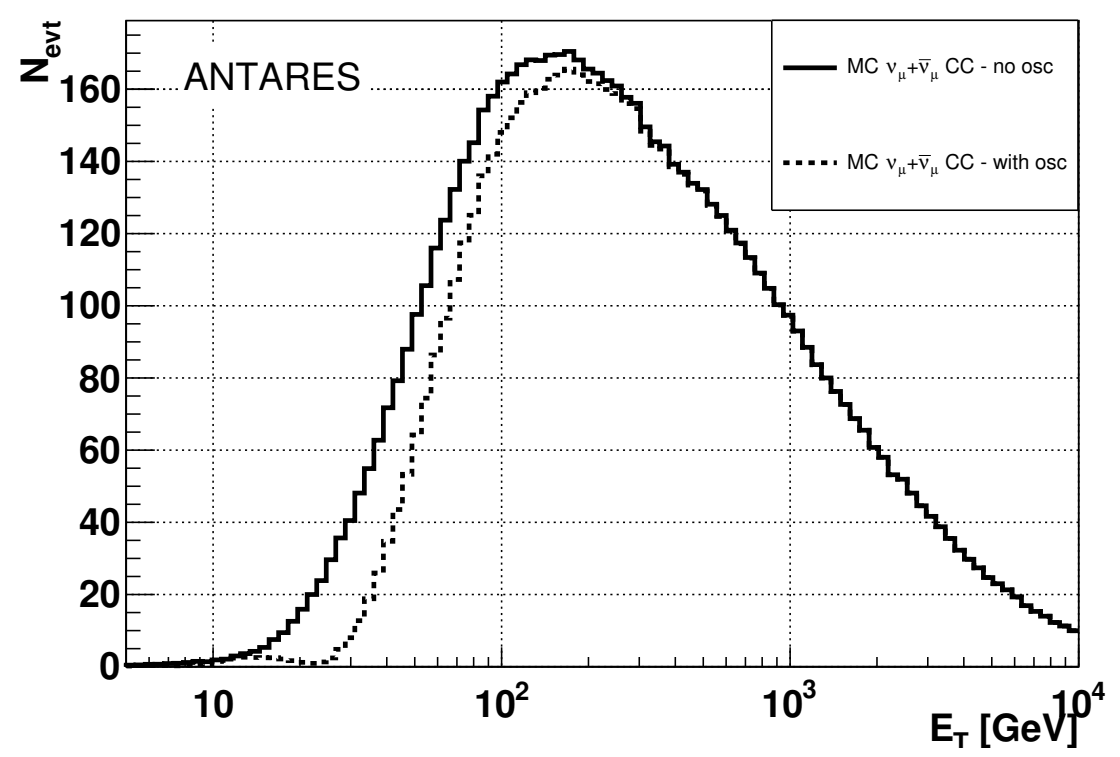

Figure 2. $\mathrm{MC}$ neutrino energy, $\mathrm{E}_{\mathrm{T}}$, for selected $\nu_{\mu}$ and $\bar{\nu}_{\mu} \mathrm{CC}$ events: assuming no oscillations (solid line) and a 2-flavour oscillation scenario with maximal mixing and $\Delta m_{32}^{2}=2.46 \times 10^{-3} \mathrm{eV}^{2}$ (dashed line).

0.5 at $25 \mathrm{GeV}$ ), the $17 \%$ branching ratio of the muonic $\tau$ decay and the resulting soft spectrum of the produced muons.

Figure 3 shows the distribution of the reduced $\chi_{\mathrm{SL}}^{2}$ for method $\mathcal{A}(\mathrm{SL})$ events where data are compared to simulated atmospheric neutrinos and background atmospheric muons. While the MC reproduces quite well the data in the signal region dominated by the neutrino signal, a disagreement between the MC expectation and data is visible for larger $\chi_{\mathrm{SL}}^{2}$. Both data and MC follow an exponential law in this region, but with different slopes. For this reason, the number of background atmospheric muons in the signal region has been determined from data itself. The distribution in figure 3 has been parameterised in the region dominated by atmospheric muons $\left(\chi_{\mathrm{SL}}^{2}>0.8\right)$ with four different exponential fits by varying the fit range. Each fit has been extrapolated into the signal region, and its corresponding integral has been computed. The mean of these integrals has been used to estimate the number of atmospheric muon background, and its uncertainty has been computed from the errors on the fitted function parameters. Summing up the results of this method for events that have been reconstructed by method $\mathcal{A}$ (SL and ML) and method $\mathcal{B}$, and combining the corresponding errors in quadrature, a total background of $740 \pm 120$ atmospheric muons has been determined. This value is subsequently used as a Gaussian prior mean value and uncertainty in the minimisation procedure. The energy and direction distribution of the atmospheric muon background has been, instead, estimated directly from MC.

After applying the event selection criteria described above on the data sample, a total of 7710 events have been selected, 1950 from method $\mathcal{A}$ (SL), 3682 from method $\mathcal{A}$ (ML) and 2078 from method $\mathcal{B}$. In figure 4 the event distribution as a function of the logarithm of the reconstructed energy, $\log _{10}\left(E_{\text {reco }} / \mathrm{GeV}\right)$, and the cosine of the reconstructed zenith 


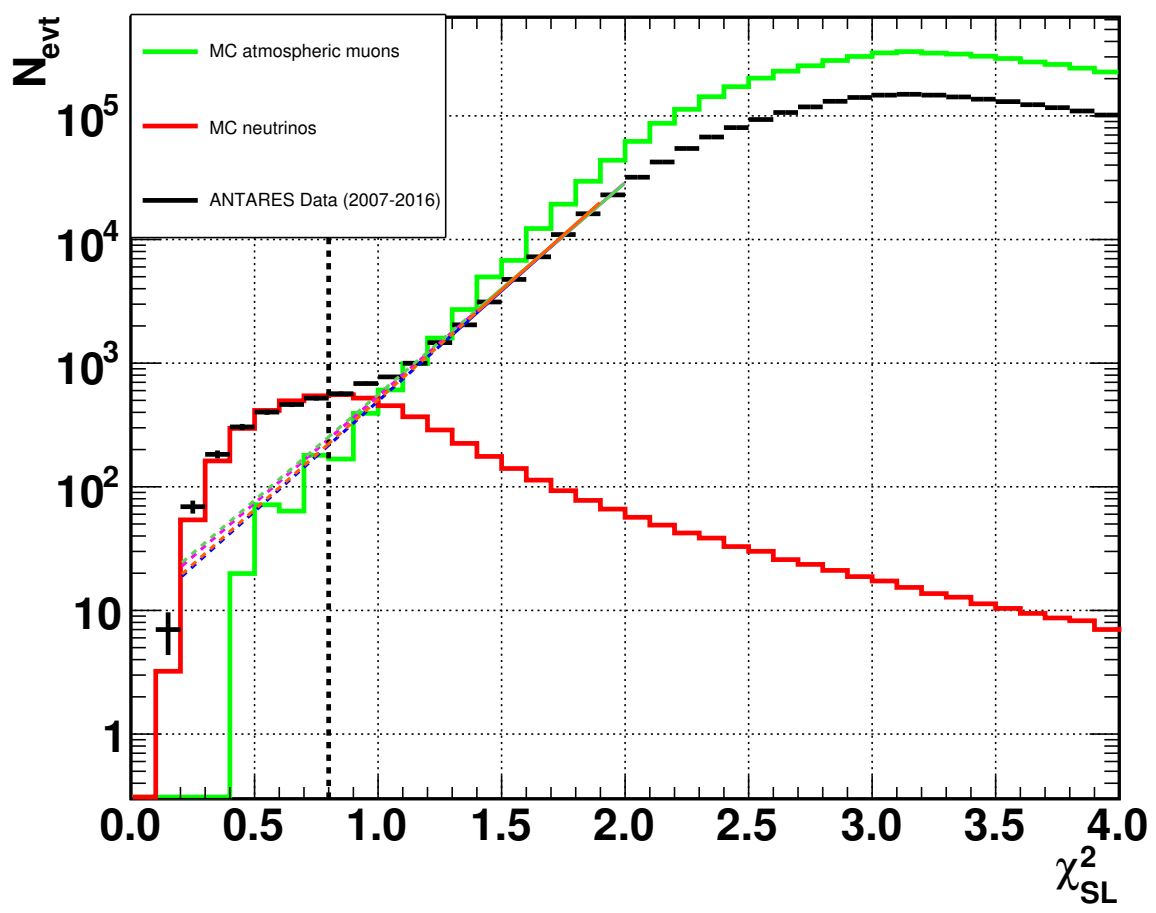

Figure 3. Distribution of reduced $\chi_{\mathrm{SL}}^{2}$ values for events which have been reconstructed by method $\mathcal{A}$ (SL). Data (black crosses) with error bars indicating the statistical uncertainty are shown together with MC neutrino events (red line) and MC atmospheric muons (green line). The dashed black line at $\chi_{\mathrm{SL}}^{2}=0.8$ indicates the value of the applied cut on this parameter. The fitted functions used to estimate the background of atmospheric muons are shown as well (solid coloured lines), together with their extrapolation into the signal region left to the cut value (dashed coloured lines, see text for details).

angle, $\cos \theta_{\text {reco }}$, is shown. The distribution of the $\mathrm{MC}$ expectation assuming no neutrino oscillation (left panel) is compared to what is observed in data (right panel). Eight bins in $\log _{10}\left(E_{\text {reco }} / \mathrm{GeV}\right)$ have been considered, seven from 1.2 to 2.0 , plus an additional underflow bin which accounts for all events with $\log _{10}\left(E_{\text {reco }} / \mathrm{GeV}\right)<1.2$; there are 17 bins in $\cos \theta_{\text {reco }}$, from 0.15 to 1.0 , the latter denoting vertically up-going events.

The final fit has been performed on the 2-dimensional histograms shown in figure 4 . The fit follows a log-likelihood approach, by minimising the function:

$$
-2 \log \mathcal{L}=2 \sum_{i, j}\left[N_{i, j}^{\mathrm{MC}}(\bar{p}, \bar{\eta})-N_{i, j}^{\mathrm{data}} \cdot \log N_{i, j}^{\mathrm{MC}}(\bar{p}, \bar{\eta})\right]+\sum_{k} \frac{\left(\eta_{k}-\left\langle\eta_{k}\right\rangle\right)^{2}}{\sigma_{\eta_{k}}^{2}},
$$

where the first sum runs over the histogram bins of $\log _{10}\left(E_{\text {reco }} / \mathrm{GeV}\right)$ and $\cos \theta_{\text {reco }}, N_{i, j}^{\text {data }}$ is the number of events in bin $(i, j)$ and $N_{i, j}^{\mathrm{MC}}(\bar{p}, \bar{\eta})$ the corresponding number of expected MC events in the same bin. This number depends on the set of oscillation parameters, $\bar{p}$, that are under investigation, as well as on the set of parameters related to systematic uncertainties, $\bar{\eta}$, as described in the next subsections. The dependency on oscillation parameters is taken into acount for CC interactions of all neutrino flavours which contribute to the final event sample. The second sum runs over the number of nuisance parameters taken into account, 

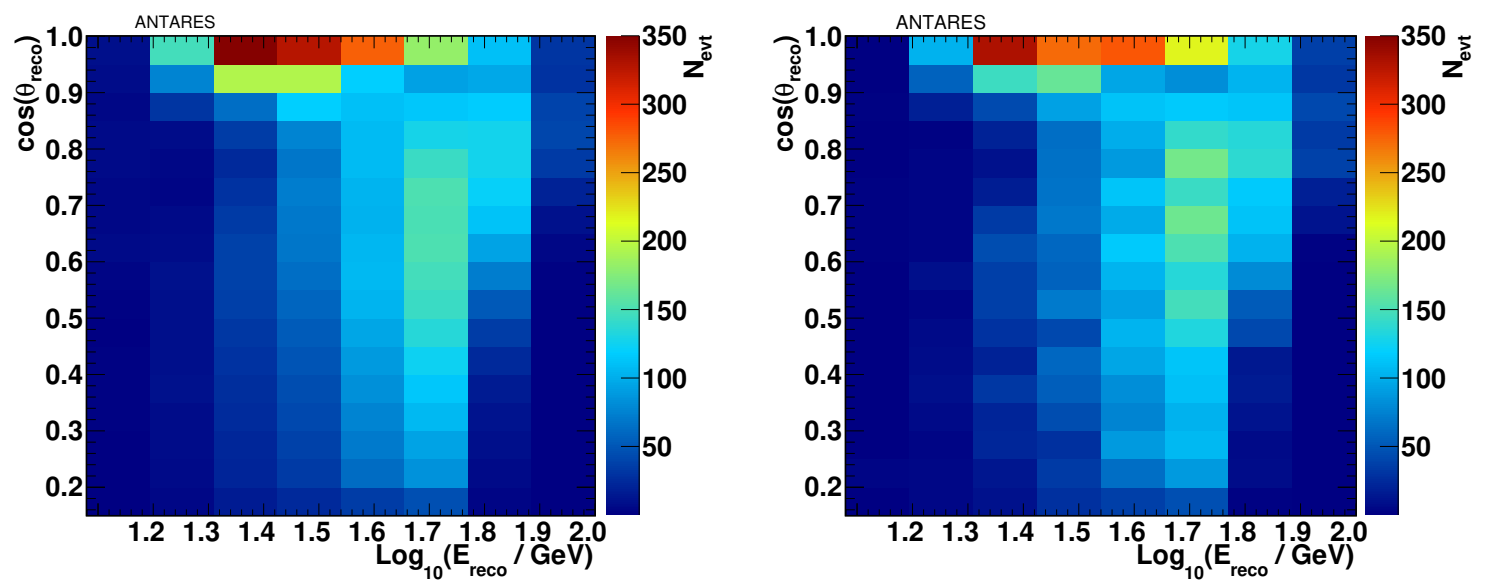

Figure 4. Number (colour scale on the right side) of selected MC events assuming no oscillation (left panel) and selected data (right panel), binned according to the logarithm of the reconstructed energy, $\log _{10}\left(E_{\text {reco }} / \mathrm{GeV}\right)$, and the reconstructed cosine of zenith, $\cos \theta_{\text {reco. }}$ The first energy bin contains all events with $\log _{10}\left(E_{\text {reco }} / \mathrm{GeV}\right)<1.2$.

$\left\langle\eta_{k}\right\rangle$ being the assumed prior of the parameter $k$, and $\sigma_{\eta_{k}}$ its uncertainty. The log-likelihood function converges to the standard $\chi^{2}$ for bins with high statistics. For bins with a small number of entries the log-likelihood is more adequate.

Since the treatment of the systematic uncertainties slightly differs between the standard atmospheric oscillation analysis and the sterile neutrino analysis, they are described separately in the following subsections.

\subsection{Treatment of systematics for the standard oscillation analysis}

The standard oscillation analysis accounts for six sources of systematic uncertainties. Three are related to the atmospheric neutrino flux. A global neutrino normalisation factor, $n_{\nu}$, which is left unconstrained during the fit, accounts for uncertainties on the total number of expected events. A variation $\Delta \gamma$ in the nominal neutrino flux spectral index has been used as additional nuisance parameter. Uncertainties on the neutrino/anti-neutrino flux ratio, $\nu / \bar{\nu}$, and on the flux asymmetry between up-going and horizontal neutrinos, $\nu_{\mathrm{up}} / \nu_{\text {hor }}$, have also been taken into account. These uncertainties [30] have been parametrised by the IceCube Collaboration [31]. Such parameterisations compute a correction on the number of expected events as a function of the neutrino energy, flavour, chirality, direction and the value of the uncertainty on the flux ratio. The two ratios considered in this analysis have been found to be strongly correlated, thus a unique nuisance parameter is considered in the fit.

An additional source of systematic uncertainty is the limited knowledge of the neutrino interaction model. At the energy of interest for this study, the cross section is dominated by deep inelastic scattering (DIS), with a smaller contribution from quasi elastic (QE) and resonant (RES) scattering. Uncertainties in the DIS cross section can be incorporated in the global flux normalisation factor $n_{\nu}$, as well as in the correction to the spectral index $\Delta \gamma$. For what concerns the QE and RES processes, dedicated studies have been performed 
with gSeaGen [32], which uses GENIE [33] to model neutrino interactions. The dominant systematic is found to be related to the axial mass for $\mathrm{CC}$ resonance neutrino production, $M_{A}$. Its default value is $1.12 \pm 0.22 \mathrm{GeV}$ [33]. By varying this parameter by $\pm 1 \sigma$, the correction with respect to the expected number of events has been computed as a function of the true neutrino energy and this parameterisation is used in the final fit.

Apart from the oscillation parameters under investigation, $\Delta m_{32}^{2}$ and $\theta_{23}$, the other oscillation parameters may play a role, but their effect is limited for this study. In particular, $\theta_{13}$ is left free in the fit but treated with a Gaussian prior at $\theta_{13}=(8.41 \pm 0.28)^{\circ}$, which is taken from a global fit [34] as well as the values of the solar neutrino parameters, which are kept fixed: $\Delta m_{21}^{2}=7.37 \times 10^{-5} \mathrm{eV}^{2}$ and $\sin ^{2} \theta_{12}=0.297$. Different values of $\delta_{\mathrm{CP}}$ have been tested at the stage of the MC sensitivity study and found to have no impact on the final result. Therefore $\delta_{\mathrm{CP}}$ is fixed at zero.

The number of atmospheric muons, $N_{\mu}$, contaminating the neutrino sample, is treated as an additional nuisance parameter. Its value and uncertainty, determined with the datadriven technique, are used as a prior.

Finally, detector and sea water related systematics have been studied as well. Dedicated MC simulations have been generated with modified OM photon detection efficiencies and a modified water absorption length, assuming a variation of $\pm 10 \%$ from the nominal value, but keeping the same wavelength dependence. The overall OM efficiency can be easily adjusted to the measured coincidence rates from ${ }^{40} \mathrm{~K}$ decays [22] which makes the chosen $10 \%$ variations a conservative benchmark value, in line with early studies performed on ANTARES OMs [17]. The water absorption length had been measured several times at the ANTARES site [35]. The different measurements, taken at two different wavelengths, vary within about $10 \%$.

The correction to the event rates, obtained by dividing the event rates from the modified MC simulation $\left(r_{\mathrm{var}}\right)$ and the one from the nominal MC simulation $\left(r_{\text {nom }}\right)$, has been computed as a function of the MC neutrino energy and zenith angle for $\nu_{\mu} \mathrm{CC}$ events, reconstructed as up-going. While no zenith-dependent effect is seen, the energy response of the detector is affected by these variations. The resulting distributions have been fitted, in the energy range $10-10^{3} \mathrm{GeV}$, with a function of the form:

$$
f_{\epsilon}\left(\mathrm{E}_{\mathrm{T}}\right)=A_{\epsilon} \cdot\left(\mathrm{E}_{\mathrm{T}} / \mathrm{E}_{0}\right)^{B_{\epsilon}},
$$

where $\mathrm{E}_{\mathrm{T}}$ is the $\mathrm{MC}$ true neutrino energy, $A_{\epsilon}, B_{\epsilon}$ are the two fitted parameters describing the effect of the modified OM photon detection efficiencies and $E_{0}=100 \mathrm{GeV}$ defines the reference energy for $A_{\epsilon}$. Figure 5 shows the distribution of the event ratios as a function of true neutrino energy, together with its parameterisation.

The effect of the modified water absorption length is described by the same functional form of eq. (5.2) using $A_{w}$ and $B_{w}$ as the corresponding fit parameters. The values of the fitted parameters $A_{\epsilon}, B_{\epsilon}, A_{w}$ and $B_{w}$ are listed in table 1 . The effects of $A_{\epsilon}$ and $A_{w}$ are taken into account in the minimisation procedure by the global normalisation factor, $n_{\nu}$, which is left unconstrained, while $B_{\epsilon}$ and $B_{w}$ are covered by the uncertainty of the prior on the spectral index, $\Delta \gamma$ (see table 2). 


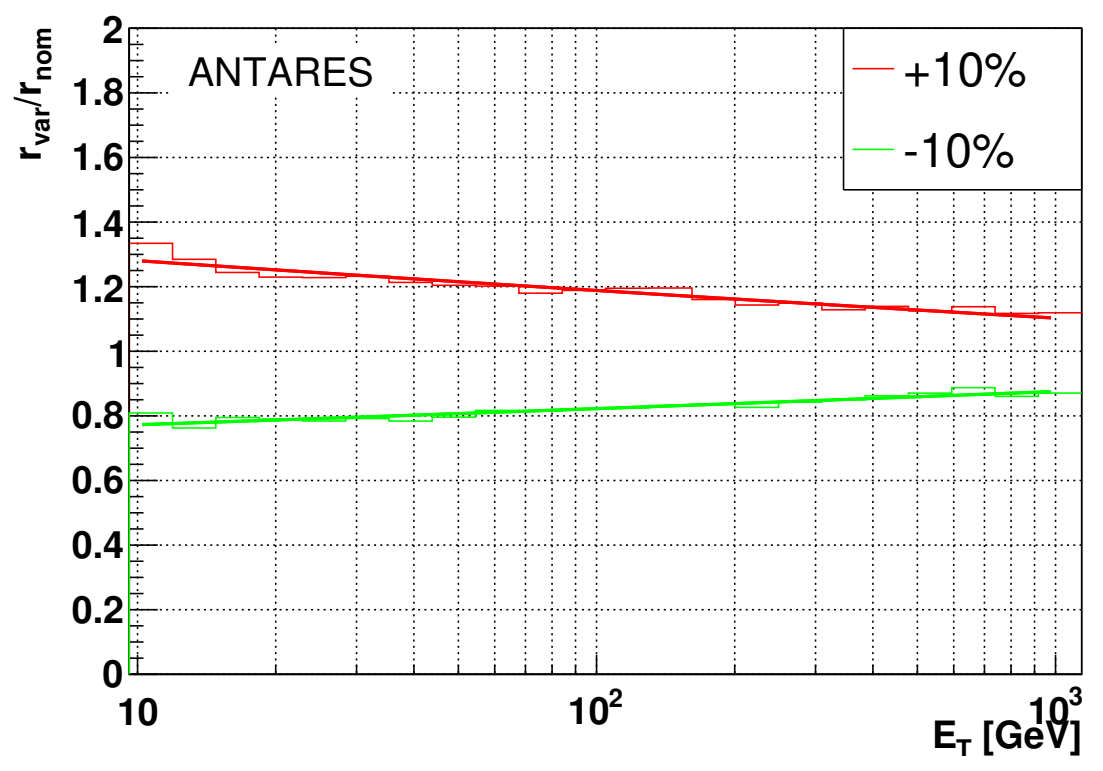

Figure 5. Expected event ratios for $\nu_{\mu} \mathrm{CC}$ events, as a function of true neutrino energy, due to a $+10 \%$ (red) and $-10 \%$ (green) variation from the nominal value of the OM photon detection efficiency.

\begin{tabular}{|c|c|c|c|c|}
\hline & $A_{\epsilon}$ & $B_{\epsilon}$ & $A_{w}$ & $B_{w}$ \\
\hline$+10 \%$ & 1.19 & -0.03 & 1.16 & -0.02 \\
$-10 \%$ & 0.82 & 0.03 & 0.92 & 0.02 \\
\hline
\end{tabular}

Table 1. Fitted values for the parameterisation of the event weight correction with a variation of $\pm 10 \%$ from the nominal value of the OM photon detection efficiency and water absorption length.

\subsection{Treatment of systematics for the sterile oscillation analysis}

For the sterile analysis, the flux as well as the cross section related systematic uncertainties are treated in the same way as described in the previous subsection.

Since the effect of a sterile neutrino would modify the oscillation pattern in a similar way as $\Delta m_{32}^{2}$ and $\theta_{23}$ do, these parameters are considered to be one of the sources of systematic uncertainty for this analysis. Both $\Delta m_{32}^{2}$ and $\theta_{23}$ are left unconstrained as recommended in [36]. The other standard oscillation parameters are treated as previously discussed.

As discussed in section 1 , the addition of a sterile neutrino in the model implies six new mixing parameters to be accounted for. The mixing angle $\theta_{14}$ and its associated phase $\delta_{14}$ have been fixed at zero, since they mainly affect the $\nu_{e}$ channel. The fast oscillations due to $\Delta m_{41}^{2} \gtrsim 0.5 \mathrm{eV}^{2}$ are unobservable due to the limited energy resolution of the detector, making $\Delta m_{41}^{2}$ not measurable. It has been kept fixed at $0.5 \mathrm{eV}^{2}$. The choice of the neutrino mass hierarchy $(\mathrm{NMH})$ as well as $\delta_{24}$ are expected to impact the result. Therefore both normal and inverted hierarchy $(\mathrm{NH} / \mathrm{IH})$ and various values of $\delta_{24}$ have been tested during the fit. Furthermore, to ensure the stability of the fit procedure, the atmospheric muon contamination has been fixed at the value found by the standard oscillation analysis. It 


\begin{tabular}{|c|c|c|}
\hline Parameter & Prior & Fit result \\
\hline$\Delta m_{32}^{2}\left[10^{-3} \mathrm{eV}^{2}\right]$ & none & $2.0_{-0.3}^{+0.4}$ \\
$\theta_{23}\left[^{\circ}\right]$ & none & $45_{-11}^{+12}$ \\
$n_{\nu}$ & none & $0.81_{-0.09}^{+0.10}$ \\
$\nu / \bar{\nu}[\sigma]$ & $0.0 \pm 1.0$ & $1.10_{-0.56}^{+0.64}$ \\
$\Delta \gamma$ & $0.00 \pm 0.05$ & $-0.003 \pm 0.036$ \\
$N_{\mu}$ & $740 \pm 120$ & $414_{-24}^{+48}$ \\
$\theta_{13}\left[^{\circ}\right]$ & $8.41 \pm 0.28$ & $8.41 \pm 0.28$ \\
$M_{A}[\sigma]$ & $0.0 \pm 1.0$ & $0.0 \pm 1.0$ \\
\hline
\end{tabular}

Table 2. Priors and fitted values obtained from the minimisation for all the parameters considered in the standard oscillation analysis.

has been verified that this choice does not lead to better constraints with respect to the case of a free muon contamination.

\section{Results}

The minimisation procedure has been done using the ROOT package Minuit2 [37], applied to the function introduced in equation (5.1). Results are presented in the following subsections, for the standard oscillation analysis and the sterile oscillation analysis, respectively.

\subsection{Results for the standard oscillation analysis}

In table 2 the complete list of all the fitted parameters for the standard oscillation analysis is shown, together with their best-fit values and their priors. Due to the high energy threshold of ANTARES this analysis is not sensitive to the NMH. The results hold for both $\mathrm{NH}$ and IH. The best-fit value is found for $\Delta m_{32}^{2}$ at $\left(2.0_{-0.3}^{+0.4}\right) \times 10^{-3} \mathrm{eV}^{2}$, which is compatible with the current world best-fit value [38]. The mixing angle $\theta_{23}$ is found to be compatible with maximal mixing within its error. The global normalisation factor for neutrinos, $n_{\nu}$, is found to be $18 \%$ lower. This value is within the atmospheric neutrino flux uncertainties and it is compatible with what was reported by other analyses [31]. A non-negligible pull is found on $\nu / \bar{\nu}$. This parameter seems to compensate for the low value of $n_{\nu}$ : this has been derived from an alternative fit, for which all nuisance parameters but $n_{\nu}$ have been fixed, to allow a more direct comparison with the result reported in [10]. Under these conditions $n_{\nu}=1.04 \pm 0.02$ is found. Concerning the spectral index correction, $\Delta \gamma$, no significant distortion from the nominal value is observed. The fitted value for the atmospheric muon contamination shows a strong pull and it is found incidentally close to the MC expectations. For both $\theta_{13}$ and $M_{A}$ the best fit values and their errors are found at the corresponding prior, which indicates no sensitivity to these parameters. This can be understood as the $\nu_{\mu}$ survival probability does not depend on $\sin \theta_{13}$ but only on $\cos \theta_{13}=0.99$ (see eq. (1.1)) whereas $M_{A}$ mainly affects neutrinos with energies below the detection threshold of ANTARES.

The distribution of the ratio between the reconstructed energy and the cosine of the reconstructed zenith is shown in figure 6 . This ratio is affected by the oscillation phe- 

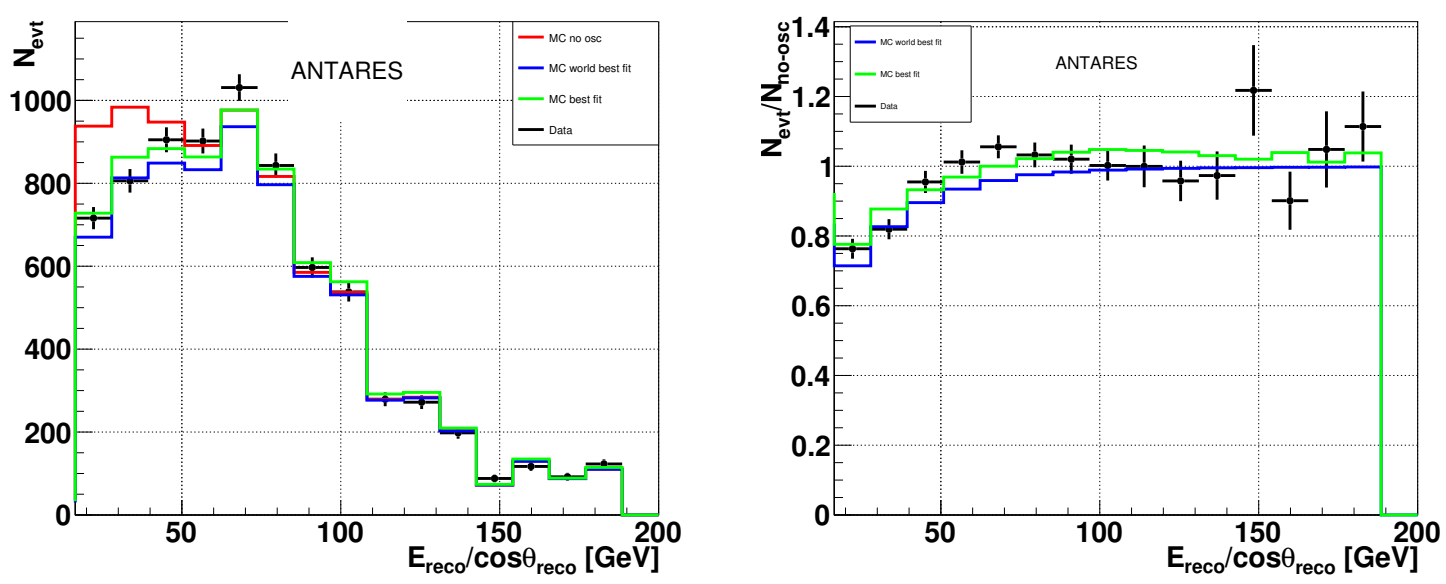

Figure 6. $E_{\text {reco }} / \cos \theta_{\text {reco }}$ distribution for data (black), MC without oscillation (red), MC assuming the world best-fit values (blue) [38] and MC assuming best-fit values of this analysis (green). The left plot shows event numbers while the right plot illustrates the event ratio with respect to the MC without oscillations.

nomenon as can be seen for the lowest values of $E_{\text {reco }} / \cos \theta_{\text {reco }}$. For comparison, also the distribution of MC assuming no neutrino oscillation, as well as the one assuming the world best-fit values [38] are shown. The latter two are calculated with all nuisance parameters at their nominal values. Such a 1D distribution does not carry the full information exploited in the fit, which is performed on the $2 \mathrm{D}$ distribution shown in figure 4 . While compatible with world data, ANTARES results seem to prefer a somewhat shallower (or energy shifted) oscillation minimum.

In figure 7 the $90 \%$ CL contour obtained in this work, in the plane of $\sin ^{2} \theta_{23}$ and $\Delta m_{32}^{2}$, is compared to those published by other experiments. The 1D projections, after profiling over the other variable, are shown as well. Confidence level contours have been computed by looping over a fine grid of values in $\Delta m_{32}^{2}$ and $\theta_{23}$ and minimising the negative log-likelihood over all the other parameters.

The non-oscillation hypothesis has been tested by performing the minimisation with a fixed null value of the oscillation parameters, and it is discarded with a significance of $4.6 \sigma$, compared to $2.3 \sigma$ in our previous analysis [10].

\subsection{Results for the sterile oscillation analysis}

In table 3 the complete list of all the fitted parameters for the sterile oscillation analysis for $\mathrm{NH}$ and $\mathrm{IH}$ is shown, together with their best-fit values and their priors. While $\theta_{24}$ is found to be compatible with zero, the best fit for $\theta_{34}$ is found at a non-zero value. This can be understood from the slight preference of the ANTARES data for a shallower oscillation dip (see discussion related to figure 6), which can be easily provided by a nonzero value of $\sin \theta_{34}$ (see figure 1 ). The non-sterile hypothesis is found at $-2 \Delta \log \mathcal{L}=4.4$ which corresponds to a 2-parameter p-value of $11 \%$. The fitted values of $\Delta m_{32}^{2}$ and $\theta_{23}$ are slightly different but consistent with respect to the ones obtained in the standard oscillation analysis. The complex phase $\delta_{24}$ is found at $180^{\circ}$. For IH instead the fit prefers $\delta_{24}=0^{\circ}$ 

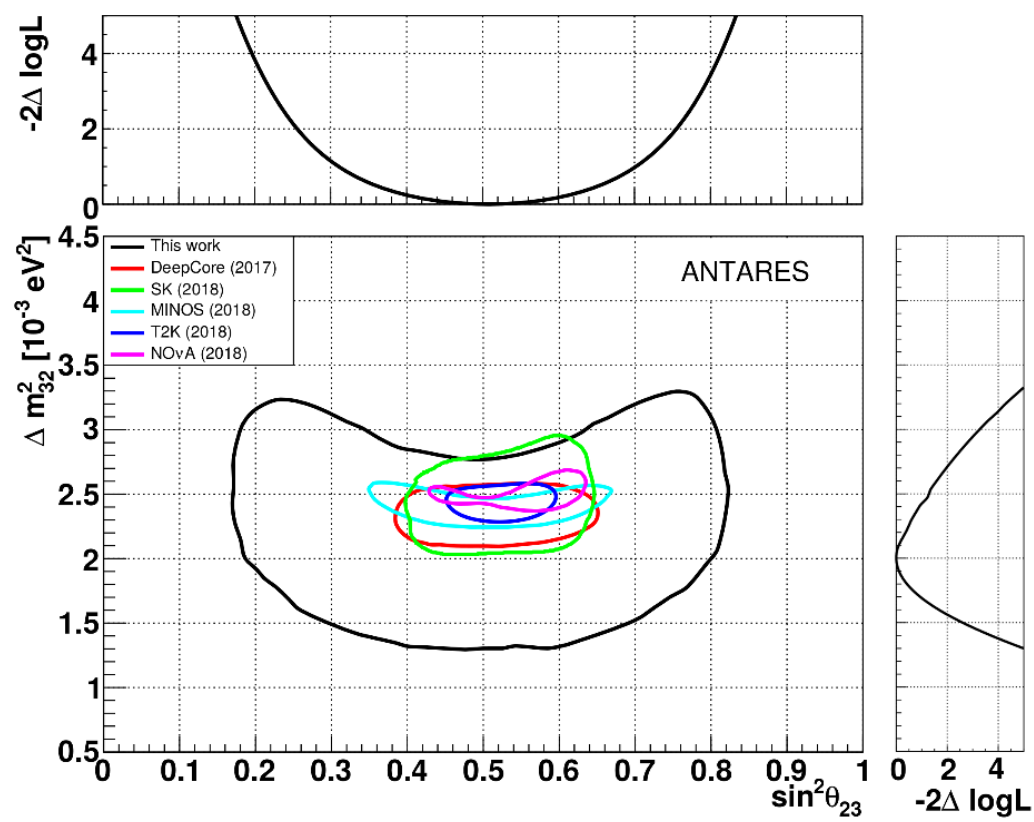

Figure 7. Contour at $90 \%$ CL in the plane of $\sin ^{2} \theta_{23}$ and $\Delta m_{32}^{2}$ obtained in this work (black line) and compared to the results by other experiments: IceCube/DeepCore (red) [31], SuperKamiokande (green) [39], NO $\nu$ A (purple) [40], T2K (blue) [41], and MINOS (light blue) [42]. The lateral plots show the 1D projections on the plane of the two oscillation parameters under study.

\begin{tabular}{|c|c|c|c|}
\hline Parameter & Prior & Fit NH & Fit IH \\
\hline$\theta_{24}\left[^{\circ}\right]$ & none & $1.5_{-5.0}^{+2.0}$ & $1.5_{-5.0}^{+2.0}$ \\
$\theta_{34}\left[^{\circ}\right]$ & none & $25.9_{-4.2}^{+5.1}$ & $25.9_{-4.2}^{+5.1}$ \\
$\delta_{24}\left[^{\circ}\right]$ & none & $180 \pm 71$ & $0 \pm 72$ \\
$n_{\nu}$ & none & $0.84_{-0.09}^{+0.10}$ & $0.84_{-0.09}^{+0.10}$ \\
$\nu / \bar{\nu}[\sigma]$ & $0.0 \pm 1.0$ & $1.07_{-0.55}^{+0.63}$ & $1.07_{-0.55}^{+0.63}$ \\
$\Delta \gamma$ & $0.00 \pm 0.05$ & $-0.011 \pm 0.036$ & $-0.011 \pm 0.036$ \\
$\Delta m_{32}^{2}\left[10^{-3} \mathrm{eV}^{2}\right]$ & none & $3.0_{-0.6}^{+0.8}$ & $-3.0_{-0.8}^{+0.6}$ \\
$\theta_{23}\left[^{\circ}\right]$ & none & $52 \pm 8$ & $52 \pm 8$ \\
$\theta_{13}\left[^{\circ}\right]$ & $8.41 \pm 0.28$ & $8.41 \pm 0.28$ & $8.41 \pm 0.28$ \\
$M_{A}[\sigma]$ & $0.0 \pm 1.0$ & $0.11_{-0.97}^{+0.93}$ & $0.11_{-0.97}^{+0.93}$ \\
\hline
\end{tabular}

Table 3. Priors and fitted values obtained from the minimisation for all the parameters considered in the sterile oscillation analysis.

with otherwise identical results, as expected from the degeneracy between $\mathrm{NMH}$ and $\delta_{24}$ (see appendix). For the other parameters a similar behaviour as for the standard oscillation analysis is observed.

Exclusion contours are built by applying Wilks' theorem. In figure 8 the resulting 90\% and $99 \%$ CL exclusion limits have been computed on a $2 \mathrm{D}$ grid in the plane of the two matrix elements, namely $\left|U_{\mu 4}\right|^{2}=\sin ^{2} \theta_{24}$ and $\left|U_{\tau 4}\right|^{2}=\sin ^{2} \theta_{34} \cos ^{2} \theta_{24}$. The exclusion limit for unconstrained $\delta_{24}$, which corresponds to both $\left[\mathrm{NH}, \delta_{24}=180^{\circ}\right]$ or $\left[\mathrm{IH}, \delta_{24}=0^{\circ}\right.$, can be 

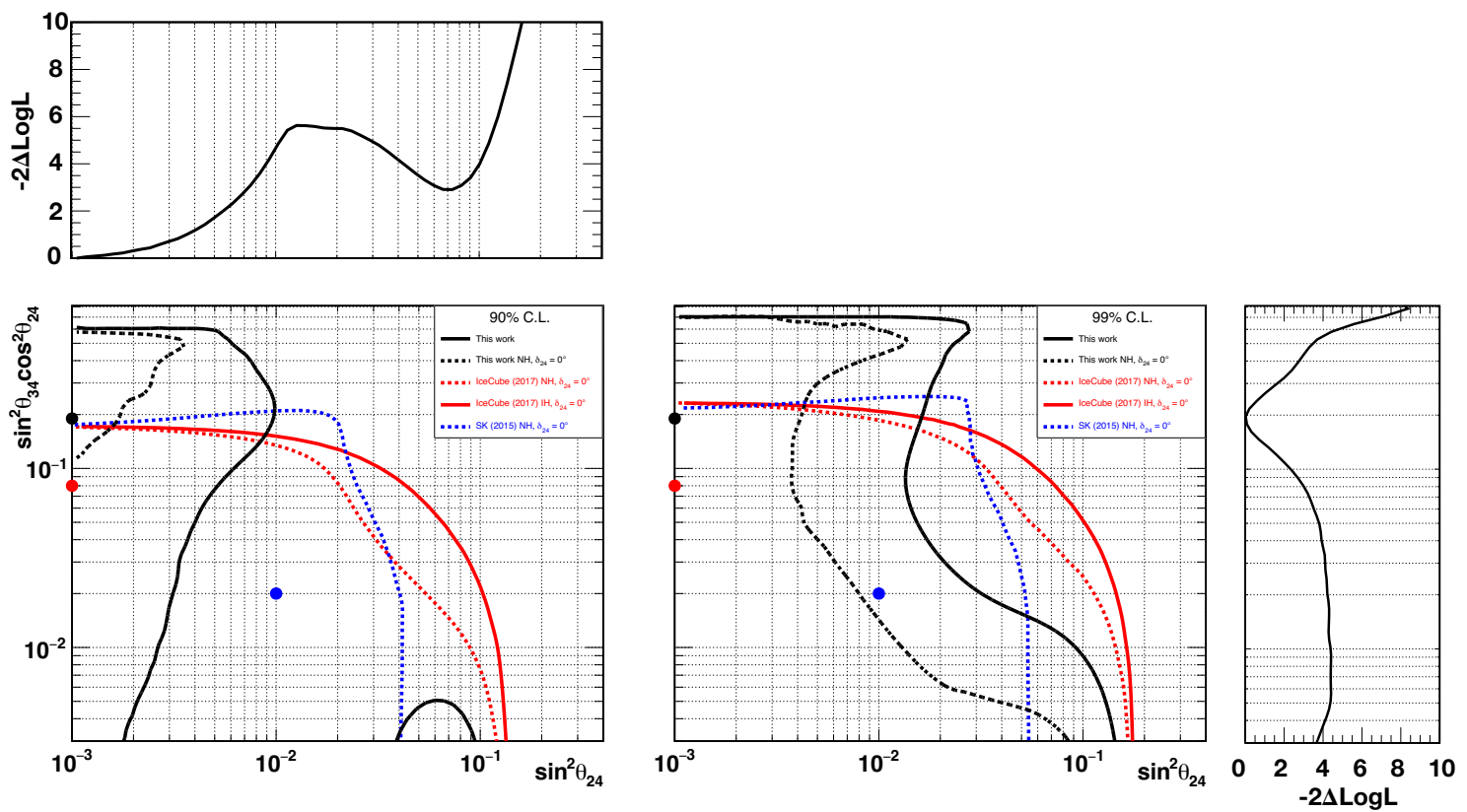

Figure 8. $90 \%$ (left) and $99 \%$ (right) CL limits for the $3+1$ neutrino model in the parameter plane of $\left|U_{\mu 4}\right|^{2}=\sin ^{2} \theta_{24}$ and $\left|U_{\tau 4}\right|^{2}=\sin ^{2} \theta_{34} \cos ^{2} \theta_{24}$ obtained in this work (black lines), and compared to the ones published by IceCube/DeepCore [16] (red) and Super-Kamiokande [15] (blue). The dashed lines are obtained for $\mathrm{NH}$ and $\delta_{24}=0^{\circ}$ while the solid lines are for an unconstrained $\delta_{24}$ (this work) or for IH and $\delta_{24}=0^{\circ}$ (IceCube/Deepcore) respectively. The coulored markers indicate the best-fit values for each experiment. The $1 \mathrm{D}$ projections after profiling over the other variable are also shown for the result of this work.

directly compared to the IceCube/DeepCore [16] (IH) limit. Also shown are limits for NH and $\delta_{\mathrm{CP}}=0^{\circ}$ which allow a direct comparison with the results from IceCube/DeepCore [16] $(\mathrm{NH})$ and Super-Kamiokande [15]. All three experiments find the best fit for $\left|U_{\tau 4}\right|^{2}$ to differ from zero. Our results exclude regions of the parameter space not yet excluded by other experiments.

The IceCube/DeepCore analysis [16] is limited to events with reconstructed energy lower than $56 \mathrm{GeV}$, while the distortion on the oscillation pattern possibly produced by the presence of a sterile neutrino would be evident also at higher reconstructed energies. The present analysis includes events with reconstructed energy up to $100 \mathrm{GeV}$. It has been verified that the ANTARES limits degrade when restricting the analysis to events with $E_{\text {reco }}<56 \mathrm{GeV}$. In this work both of the standard atmospheric oscillation parameters $\Delta m_{32}^{2}$ and $\sin ^{2}\left(2 \theta_{23}\right)$ are left unconstrained in line with the IceCube/DeepCore analysis [16].

After profiling over the other variable, the following limits on the two matrix elements can be derived:

$$
\begin{aligned}
& \left|U_{\mu 4}\right|^{2}<0.007(0.13) \text { at } 90 \%(99 \%) \mathrm{CL}, \\
& \left|U_{\tau 4}\right|^{2}<0.40(0.68) \text { at } 90 \%(99 \%) \mathrm{CL} .
\end{aligned}
$$




\section{Conclusions}

Ten years of ANTARES data have been analysed to provide a measurement of the atmospheric neutrino oscillation parameters. The analysis chain has been optimised with respect to our previously published study, by combining two track reconstruction algorithms and introducing a more elaborate treatment of various sources of systematic uncertainties. The results, $\Delta m_{32}^{2}=\left(2.0_{-0.3}^{+0.4}\right) \times 10^{-3} \mathrm{eV}^{2}$ and $\theta_{23}=\left(45_{-11}^{+12}\right)^{\circ}$, are consistent with what has been published by other experiments. The non-oscillation hypothesis is discarded with a significance of $4.6 \sigma$.

Exploiting the same analysis chain and the same data set, a further study has allowed to constrain, for the first time with ANTARES, the parameter space of the $3+1$ neutrino model, which foresees the existence of one sterile neutrino. ANTARES excludes values of the parameter space not yet excluded by other experiments.

\section{Acknowledgments}

The authors acknowledge the financial support of the funding agencies: Centre National de la Recherche Scientifique (CNRS), Commissariat à l'énergie atomique et aux énergies alternatives (CEA), Commission Européenne (FEDER fund and Marie Curie Program), Institut Universitaire de France (IUF), IdEx program and UnivEarthS Labex program at Sorbonne Paris Cité (ANR-10-LABX-0023 and ANR-11-IDEX-0005-02), Labex OCEVU (ANR-11-LABX-0060) and the A*MIDEX project (ANR-11-IDEX-0001-02), Région Île-deFrance (DIM-ACAV), Région Alsace (contrat CPER), Région Provence-Alpes-Côte d'Azur, Département du Var and Ville de La Seyne-sur-Mer, France; Bundesministerium für Bildung und Forschung (BMBF), Germany; Istituto Nazionale di Fisica Nucleare (INFN), Italy; Nederlandse organisatie voor Wetenschappelijk Onderzoek (NWO), the Netherlands; Council of the President of the Russian Federation for young scientists and leading scientific schools supporting grants, Russia; Executive Unit for Financing Higher Education, Research, Development and Innovation (UEFISCDI), Romania; Ministerio de Economía y Competitividad (MINECO): Plan Estatal de Investigación (refs. FPA2015-65150-C3-1-P, -2-P and -3-P, (MINECO/FEDER)), Severo Ochoa Centre of Excellence and Red Consolider MultiDark (MINECO), and Prometeo and Grisolía programs (Generalitat Valenciana), Spain; Ministry of Higher Education, Scientific Research and Professional Training, Morocco. We also acknowledge the technical support of Ifremer, AIM and Foselev Marine for the sea operation and the CC-IN2P3 for the computing facilities. We would like to thank J. Coelho for enlightening discussions on matter effects for sterile neutrinos.

\section{A Sterile neutrinos and matter effects}

For the analysis presented in this paper, oscillation probabilities are evaluated with the software package OscProb [8]. However, in this appendix some common approximations are applied, to derive analytical formulae. These are NOT used for the analysis itself but allow to get a better understanding of the interplay between different parameters. The $\nu_{\mu}$ 
survival probability in vacuum in the $3+1$ model can be simplified with the following two hypotheses [15, 43]: first, it is assumed, that the first generation decouples completely, i.e. $\Delta m_{21}^{2}=0$ and $\theta_{12}=\theta_{13}=0$; second, fast wiggles due to oscillations involving $m_{4}$ are assumed to be unobservable, i.e. $\sin ^{2}\left(\Delta m_{4 i}^{2} L / 4 E\right)=1 / 2$ for all $i$. This yields

$$
P_{\nu_{\mu} \rightarrow \nu_{\mu}}=\left(1-\left|U_{\mu 4}\right|^{2}\right)^{2} P_{\mu \mu}^{(3)}+\left|U_{\mu 4}\right|^{4}
$$

with $P_{\mu \mu}^{(3)}$ the $\nu_{\mu}$ survival probability in the 3 -flavour scheme, i.e. without additional sterile neutrinos. Only $\left|U_{\mu 4}\right|^{2}=\sin ^{2} \theta_{24}$ can be probed in this scheme, which is applied in most accelerator based $\nu_{\mu}$ disappearance analyses. However, when analysing atmospheric neutrinos, matter effects cannot be neglected. An analytical formalism is developped in eqs. (4.13)-(4.25) of [15]. In eq. (4.13), a complex phase is present in the non-diagonal term of the matrix, which is neglected, i.e. set to zero, in subsequent steps. If instead this phase is kept, $\sin 2 \theta_{s}$ in eq. (4.16) acquires an extra term $\exp (-i \delta)$.

$$
\begin{aligned}
\sin 2 \theta_{s} & =\frac{2 \sqrt{\left|U_{\mu 4}\right|^{2}\left|U_{\tau 4}\right|^{2}\left(1-\left|U_{\mu 4}\right|^{2}-\left|U_{\tau 4}\right|^{2}\right)}}{\left(1-\left|U_{\mu 4}\right|^{2}\right)\left(\left|U_{\mu 4}\right|^{2}+\left|U_{\tau 4}\right|^{2}\right)} e^{-i \delta}, \\
\cos 2 \theta_{s} & =\frac{\left|U_{\tau 4}\right|^{2}-\left|U_{\mu 4}\right|^{2}\left(1-\left|U_{\mu 4}\right|^{2}-\left|U_{\tau 4}\right|^{2}\right)}{\left(1-\left|U_{\mu 4}\right|^{2}\right)\left(\left|U_{\mu 4}\right|^{2}+\left|U_{\tau 4}\right|^{2}\right)}
\end{aligned}
$$

This in turn modifies eqs. (4.18) and eq. (4.19):

$$
\begin{aligned}
E_{m}^{2} & =A_{32}^{2}+A_{s}^{2}+2 A_{32} A_{s}\left(\sin 2 \theta_{23}\left|\sin 2 \theta_{s}\right| \cos \delta+\cos 2 \theta_{23} \cos 2 \theta_{s}\right) \\
\sin 2 \theta_{m} & =\frac{1}{E_{m}} \sqrt{A_{32}^{2} \sin ^{2} 2 \theta_{23}+A_{s}^{2}\left|\sin 2 \theta_{s}\right|^{2}+2 A_{32} A_{s} \sin 2 \theta_{23}\left|\sin 2 \theta_{s}\right| \cos \delta} .
\end{aligned}
$$

For $\delta=0$ the original expressions from [15] are reproduced. With $A_{32}=\Delta m_{32}^{2} / E_{\nu}$ and $A_{s}=\frac{\sqrt{2}}{2} G_{F} N_{n}\left(\left|U_{\mu 4}\right|^{2}+\left|U_{\tau 4}\right|^{2}\right) / 2\left(G_{F}\right.$ the Fermi constant and $N_{n}$ the neutron density) the $\nu_{\mu}$ survival probability in matter is fully defined and can be written equivalently to eq. (A.1) (see also eq. (4.23) of [15]):

$$
P_{\nu_{\mu} \rightarrow \nu_{\mu}}=\left(1-\left|U_{\mu 4}\right|^{2}\right)^{2}\left(1-\sin ^{2} 2 \theta_{m} \sin ^{2}\left(E_{m} L\right)\right)+\left|U_{\mu 4}\right|^{4},
$$

which describes well all features shown in figure 1. The impact of the CP-phase $\delta$ disappears when either $\left|U_{\mu 4}\right|^{2}=0$ or $\left|U_{\tau 4}\right|^{2}=0$, which leads to $\sin 2 \theta_{s}=0$. Further, $\delta \rightarrow \delta+\pi$ is completely degenerate with changing the mass hierarchy, i.e. swapping the sign of $A_{32}$ if either $\cos 2 \theta_{23}=0$ or $\cos 2 \theta_{s}=0$. Deviation from maximal mixing in $\theta_{23}$ or from the symmetry between $\left|U_{\mu 4}\right|^{2}$ and $\left|U_{\tau 4}\right|^{2}$ defining $\theta_{s}$ breaks this degeneracy. The impact of the neutrino mass hierarchy on the $\nu_{\mu}$ survival probability in matter had been pointed out already in [44], while the influence of complex phases is also discussed in [45].

Open Access. This article is distributed under the terms of the Creative Commons Attribution License (CC-BY 4.0), which permits any use, distribution and reproduction in any medium, provided the original author(s) and source are credited. 


\section{References}

[1] Z. Maki, M. Nakagawa and S. Sakata, Remarks on the unified model of elementary particles, Prog. Theor. Phys. 28 (1962) 870 [inSPIRE].

[2] B. Pontecorvo, Neutrino experiments and the problem of conservation of leptonic charge, Sov. Phys. JETP 26 (1968) 984 [INSPIRE].

[3] V.N. Gribov and B. Pontecorvo, Neutrino astronomy and lepton charge, Phys. Lett. B 28 (1969) 493 [INSPIRE].

[4] Particle Data Group collaboration, Review of particle physics, Phys. Rev. D 98 (2018) 030001 [INSPIRE].

[5] L. Wolfenstein, Neutrino oscillations in matter, Phys. Rev. D 17 (1978) 2369 [InSPIRE].

[6] S.P. Mikheyev and A.Y. Smirnov, Resonance amplification of oscillations in matter and spectroscopy of solar neutrinos, Sov. J. Nucl. Phys. 42 (1985) 913 [INSPIRE].

[7] S.P. Mikheev and A.Y. Smirnov, Resonant amplification of neutrino oscillations in matter and solar neutrino spectroscopy, Nuovo Cim. C 9 (1986) 17 [INSPIRE].

[8] J. Coelho, OscProb, https://github.com/joaoabcoelho/OscProb/.

[9] ANTARES collaboration, ANTARES: the first undersea neutrino telescope, Nucl. Instrum. Meth. A 656 (2011) 11 [arXiv:1104.1607] [INSPIRE].

[10] ANTARES collaboration, Measurement of atmospheric neutrino oscillations with the ANTARES neutrino telescope, Phys. Lett. B 714 (2012) 224 [arXiv:1206.0645] [INSPIRE].

[11] LSND collaboration, Evidence for neutrino oscillations from the observation of $\bar{\nu}_{e}$ appearance in a $\bar{\nu}_{\mu}$ beam, Phys. Rev. D 64 (2001) 112007 [hep-ex/0104049] [INSPIRE].

[12] MiniBoonE and SciBooNE collaborations, Dual baseline search for muon antineutrino disappearance at $0.1 \mathrm{eV}^{2}<\Delta \mathrm{m}^{2}<100 \mathrm{eV}^{2}$, Phys. Rev. D 86 (2012) 052009 [arXiv: 1208.0322] [INSPIRE].

[13] Aleph, DELPHI, L3, OPAL, SLD, LEP Electroweak Working Group, SLD Electroweak Group and SLD Heavy Flavour Group collaborations, Precision electroweak measurements on the $Z$ resonance, Phys. Rept. 427 (2006) 257 [hep-ex/0509008] [INSPIRE].

[14] MINOS+ collaboration, Search for sterile neutrinos in MINOS and MINOS+ using a two-detector fit, Phys. Rev. Lett. 122 (2019) 091803 [arXiv: 1710.06488] [INSPIRE].

[15] SUPER-KAmiOKANDE collaboration, Limits on sterile neutrino mixing using atmospheric neutrinos in Super-Kamiokande, Phys. Rev. D 91 (2015) 052019 [arXiv:1410.2008] [INSPIRE].

[16] ICECuBE collaboration, Search for sterile neutrino mixing using three years of IceCube DeepCore data, Phys. Rev. D 95 (2017) 112002 [arXiv:1702.05160] [InSPIRE].

[17] ANTARES collaboration, The ANTARES optical module, Nucl. Instrum. Meth. A 484 (2002) 369 [astro-ph/0112172] [INSPIRE].

[18] ANTARES collaboration, Performance of the front-end electronics of the ANTARES neutrino telescope, Nucl. Instrum. Meth. A 622 (2010) 59 [arXiv:1007.2549] [InSPIRE].

[19] ANTARES collaboration, The data acquisition system for the ANTARES neutrino telescope, Nucl. Instrum. Meth. A 570 (2007) 107 [astro-ph/0610029] [INSPIRE]. 
[20] B. Bakker, Trigger studies for the Antares and KM3NeT neutrino telescopes, Master's Thesis, University of Amsterdam (2011).

[21] ANTARES collaboration, The Run-by-Run Monte Carlo simulation for the ANTARES experiment, EPJ Web Conf. 116 (2016) 02002 [INSPIRE].

[22] ANTARES collaboration, Long-term monitoring of the ANTARES optical module efficiencies using ${ }^{40} \mathrm{~K}$ decays in sea water, Eur. Phys. J. C 78 (2018) 669 [arXiv: 1805.08675] [INSPIRE].

[23] D. Bailey, Monte Carlo tools and analysis methods for understanding the ANTARES experiment and predicting its sensitivity to dark matter, Ph.D. Thesis, Oxford University (2002).

[24] M. Honda, M. Sajjad Athar, T. Kajita, K. Kasahara and S. Midorikawa, Atmospheric neutrino flux calculation using the NRLMSISE-00 atmospheric model, Phys. Rev. D 92 (2015) 023004 [arXiv: 1502.03916] [INSPIRE].

[25] G. Carminati, M. Bazzotti, A. Margiotta and M. Spurio, Atmospheric MUons from PArametric formulas: a fast GEnerator for neutrino telescopes (MUPAGE), Comput. Phys. Commun. 179 (2008) 915 [arXiv:0802.0562] [INSPIRE].

[26] Application Software Group collaboration, GEANT 3: a detector description and simulation tool, CERN Program Library Long Writeup W5013 (1995).

[27] ANTARES collaboration, A fast algorithm for muon track reconstruction and its application to the ANTARES neutrino telescope, Astropart. Phys. 34 (2011) 652 [arXiv:1105.4116] [INSPIRE].

[28] E. Visser, Neutrinos from the Milky Way, Ph.D. Thesis, Universiteit Leiden (2015).

[29] C. Patrignani et al., The review of particle physics, Chin. Phys. C 40 (2016) 100001.

[30] G.D. Barr, T.K. Gaisser, S. Robbins and T. Stanev, Uncertainties in atmospheric neutrino fluxes, Phys. Rev. D 74 (2006) 094009 [astro-ph/0611266] [INSPIRE].

[31] ICECuBE collaboration, Measurement of atmospheric neutrino oscillations at 6-56 GeV with IceCube DeepCore, Phys. Rev. Lett. 120 (2018) 071801 [arXiv:1707.07081] [INSPIRE].

[32] ANTARES and KM3NET collaborations, gSeaGen: a GENIE-based code for neutrino telescopes, EPJ Web Conf. 116 (2016) 08001 [arXiv:1602.00501] [INSPIRE].

[33] C. Andreopoulos et al., The GENIE Neutrino Monte Carlo Generator: physics and user manual, arXiv: 1510.05494 [INSPIRE].

[34] F. Capozzi, E. Lisi, A. Marrone, D. Montanino and A. Palazzo, Neutrino masses and

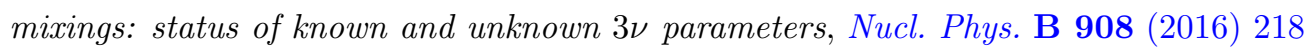
[arXiv: 1601.07777] [INSPIRE].

[35] ANTARES collaboration, Transmission of light in deep sea water at the site of the ANTARES neutrino telescope, Astropart. Phys. 23 (2005) 131 [astro-ph/0412126] [INSPIRE].

[36] S.K. Agarwalla, S.S. Chatterjee and A. Palazzo, Signatures of a light sterile neutrino in T2HK, JHEP 04 (2018) 091 [arXiv: 1801.04855] [INSPIRE].

[37] R. Brun and F. Rademakers, ROOT: an object oriented data analysis framework, Nucl. Instrum. Meth. A 389 (1997) 81 [INSPIRE]. 
[38] P.F. de Salas, D.V. Forero, C.A. Ternes, M. Tortola and J.W.F. Valle, Status of neutrino oscillations 2018: $3 \sigma$ hint for normal mass ordering and improved CP sensitivity, Phys. Lett. B 782 (2018) 633 [arXiv:1708.01186] [INSPIRE].

[39] Super-Kamiokande collaboration, Atmospheric neutrino oscillation analysis with external constraints in Super-Kamiokande I-IV, Phys. Rev. D 97 (2018) 072001 [arXiv:1710.09126] [INSPIRE].

[40] M. Sanchez, NOvA results and prospects, https://doi.org/10.5281/zenodo.1286758 (2018).

[41] M. Wascko, T2K status, results, and plans, https://doi.org/10.5281/zenodo.1286752 (2018).

[42] A. Aurisano, Recent results from MINOS and MINOS+, https://doi.org/10.5281/zenodo.1286760 (2018).

[43] M. Maltoni and T. Schwetz, Sterile neutrino oscillations after first MiniBooNE results, Phys. Rev. D 76 (2007) 093005 [arXiv:0705.0107] [InSPIRE].

[44] S. Razzaque and A.Y. Smirnov, Searches for sterile neutrinos with IceCube DeepCore, Phys. Rev. D 85 (2012) 093010 [arXiv:1203.5406] [INSPIRE].

[45] M. Blennow, E. Fernandez-Martinez, J. Gehrlein, J. Hernandez-Garcia and J. Salvado, IceCube bounds on sterile neutrinos above $10 \mathrm{eV}$, Eur. Phys. J. C 78 (2018) 807 [arXiv: 1803.02362] [INSPIRE]. 